\title{
Data Mining for Material Feeding Optimization of Printed Circuit Board Template Production
}

\author{
Shengping Lv $\mathbb{D}^{1,2}$ Binbin Zheng, ${ }^{1}$ Hoyeol Kim, ${ }^{2}$ and Qiangsheng Yue \\ ${ }^{1}$ College of Engineering, South China Agricultural University, Guangzhou 510642, China \\ ${ }^{2}$ Department of Industrial, Manufacturing and Systems Engineering, Texas Tech University, Lubbock, TX 79409, USA \\ Correspondence should be addressed to Shengping Lv; lvshengping@scau.edu.cn
}

Received 7 November 2017; Accepted 18 February 2018; Published 1 April 2018

Academic Editor: Ephraim Suhir

Copyright (c) 2018 Shengping Lv et al. This is an open access article distributed under the Creative Commons Attribution License, which permits unrestricted use, distribution, and reproduction in any medium, provided the original work is properly cited.

\begin{abstract}
Improving the accuracy of material feeding for printed circuit board (PCB) template orders can reduce the overall cost for factories. In this paper, a data mining approach based on multivariate boxplot, multiple structural change model (MSCM), neighborhood component feature selection (NCFS), and artificial neural networks (ANN) was developed for the prediction of scrap rate and material feeding optimization. Scrap rate related variables were specified and 30,117 samples of the orders were exported from a PCB template production company. Multivariate boxplot was developed for outlier detection. MSCM was employed to explore the structural change of the samples that were finally partitioned into six groups. NCFS and ANN were utilized to select scrap rate related features and construct prediction models for each group of the samples, respectively. Performances of the proposed model were compared to manual feeding, $\mathrm{ANN}$, and the results indicate that the approach exhibits obvious superiority to the other two methods by reducing surplus rate and supplemental feeding rate simultaneously and thereby reduces the comprehensive cost of raw material, production, logistics, inventory, disposal, and delivery tardiness compensation.
\end{abstract}

\section{Introduction}

Printed circuit board (PCB) is found in practically all electrical and electronic equipment (EEE), being the base of the electronics industry [1]. Due to increased competition and market volatility, demand for highly individualized products promotes a rapid growth of $\mathrm{PCB}$ orders designed with many specialized features but short delivery time $[2,3]$. Customeroriented small batch production is always employed by a factory with lots of PCB template orders, which is different from mass production, and therefore causes companies to face serious challenges. Optimization of material feeding is one of the critical problems.

The scrap rate and material feeding area of each $\mathrm{PCB}$ template order are difficult to be accurately determined in advance of the production. Many factories undergo the violent fluctuation in both surplus rate and supplemental feeding rate due to empirical manual feeding in practice by heavily depending on their experience and knowledge. Individualized surplus template products can be placed in inventory or directly destroyed while frequent material feeding brings supplemental production cost and delivery tardiness compensation. This motivates us to explore the pattern of historical orders through data mining (DM) approach to facilitate more reasonable material feeding for the orders automatically and therefore reduce the comprehensive cost caused by excessive or underestimated material feeding before production.

The general process of DM also known as knowledge discovery in databases (KDD) includes problem clarification, data preparation, preprocessing, DM in the narrow sense, and interpretation and evaluation of results [4]. DM in the narrow sense as a step in the KDD process consists of applying data analysis and particular discovery algorithm within an acceptable computational efficiency limit [4]. DM tasks can be classified into descriptive and predictive two groups [4, 5]. Descriptive function of DM mainly aims to explore the potential or recessive rules, characteristics, and relationships (dependency, similarity, etc.) that exist in the data, such as generalization, association, sequence pattern mining, and clustering [4-8]. As to the predictive functions of DM, they are usually selected to analyze the relevant trends of 
the data or the relevant laws to predict the future state. It includes classification, prediction, time series, and analysis [4-8]. To achieve these goals, DM solutions employ a wide variety of enabling techniques and specific mining techniques to both predict and describe interpretable and valuable information $[4,5,8-10]$. The enabling techniques mainly refer to the methods for data cleaning, data integration, data transformation, and data reduction that can support the implementation of DM in the narrow sense, while specific mining techniques, like regression, support vector machine, and artificial neural network (ANN), are the approaches used to explore useful knowledge from massive data $[4,9]$. The scrap rate prediction and material feeding optimization of PCB template production can be taken as an application of predictive function of DM; and the specification of scrap rate related features, identifying features which affect scrap rate significantly and related mining techniques, should be carefully studied. Moreover, many features (e.g., required panel) have structural change influence for the scrap rate according to empirical knowledge, and therefore the enabling and mining techniques, interpretation, and evaluation steps in DM should also be adjusted accordingly.

The details of enabling techniques, DM applications for different manufacturing task and different manufacturing industry, patterns in the use of specific mining techniques, application performance, and software used in these applications have been widely studied, and one can refer to [4-10] for comprehensive review. Electronic product manufacturing industries also exploited several DM methods with the purpose of summarization, clustering, association, classification, prediction, and so on $[5,8]$. And many are closely related to PCB manufacturing industry. Tseng et al. employed Kohonen neural networks, decision tree (DT), and multiple regression to improve accuracy of work hours estimation based on the PCB design data, and the performance clearly exceeds the conventional method of regression equations [11]. Tsai et al. developed three hybrid approaches including ANN-genetic algorithm (GA), fuzzy logic-Taguchi, and regression analysisresponse surface methodology (RSM) to predict the volume and centroid offset two responses and optimize parameters for the micro ball grid array (BGA) packages during the stencil printing process (SPP) for components assembly on $\mathrm{PCB}$, and the confirmation experiments show that the proposed fuzzy logic-based Taguchi method outperforms the other two methods in terms of the signal-to-noise ratios and process capability index $[12,13]$. Some other approaches, like support vector regression (SVR) and mixed-integer linear programming, have also been developed for the parameter optimization of SPP [14]. Haneda et al. [15] employed variable cluster analysis and $K$-means approach to help engineers determine appropriate drilling condition and parameter for PCB manufacturing. DM-based defect (faults) diagnosis or quality control during manufacturing has also been widely studied [5, 7], and many algorithms like adaptive genetic algorithm-ANN [16] and DT [17] have been developed for the defect (faults) diagnosis of PCB manufacturing.

The marketing and sales is another widely investigated direction of DM application in PCB industry. Success in forecasting and analyzing sales for given goods or services can mean the difference between profit and loss for an accounting period [18]. Many DM-based methods like $K$-mean cluster and fuzzy neural network, fuzzy case-based reasoning, and weighted evolving fuzzy neural network have been developed by Chang et al. [19-21] to select a combination of key factors which have the greatest influence on PCB marketing and then forecasts the future PCB sales. Tavakkoli et al. [22] combined SVR, Bat metaheuristic, and Taguchi method to predict the future PCB sales, and performance comparison indicates that the accuracy of the proposed hybrid model is better than the GA-SVR, particle swarm optimization-SVR, and classical SVR. Hadavandi et al. hybridize fuzzy logic with GA and $K$ means to extract useful information patterns from sales data, and results show that the proposed approach outperforms the other previous approaches [18].

However, the quality related research for PCB manufacturing mainly focuses on one operation of the manufacturing process for the purpose of yield improvement [12-15], and there are few studies on material feeding optimization especially for PCB production using DM mechanism to the best of our knowledge. Meanwhile, the change structure of the studied problem and corresponding change of relevant features have seldom been considered during the mining procedure. Meanwhile, ANN-based approach, as a most frequently used DM method that will also be employed in the study, tries to exploit nonlinear patterns in different problems demonstrating reasonable results; however, problem divided into different subproblems according to structural change always requires different $\mathrm{ANN}$ architecture and different learned link weights based on different input features, while this is difficult to learn by the ANN without reasonable preprocessing.

In this paper, a data mining approach (MSCM-ANN) is presented to establish the scrap rate prediction model and optimize material feeding of PCB orders considering the structural change influence based on the use of multivariate boxplot, multiple structural change model (MSCM), neighborhood component feature selection (NCFS), and ANN. The comparison of MSCM-ANN to ANN and manual feeding will be conducted to verify the performance of the proposed approaches. The rest of the paper is organized as follows. In Section 2, variables specification and sample data are described. Methodology, including multivariate boxplot, MSCM, NCFS, ANN, and performance indicators, is presented in Section 3, followed by experimental results and discussion in Section 4. Lastly, conclusions are drawn in Section 5.

\section{Variables and Sample}

The data used in this study were collected from Guangzhou FastPrint Technology Co., Ltd. A total of 56 variables inherited from enterprise resource planning system combined with the derived variables were selected and specified in Table 1, in which variables 40 to 56 are the statistic results of manual feeding adopted by FastPrint. The unit in a panel, required quantity/panel/area, and delivery unit area can not only be taken as statistic items, but also feature candidates for MSCM-ANN and ANN model establishment.

Set and unit are two types of delivery unit, whereas panel is production unit that will be partitioned into either set 
TABLE 1: Variables specification.

\begin{tabular}{|c|c|c|c|c|}
\hline Number & Variable name & Symbol & Description & Value range \\
\hline \multicolumn{5}{|c|}{ Overall characteristics } \\
\hline 1 & PCB thickness & $P t$ & Thickness of the ordered PCB & $0.3-8$ \\
\hline 2 & Layer number & $L n$ & Number of copper layers & $4-20$ \\
\hline 3 & Rogers material & Ro & Whether substrate material is Rogers & $0 / 1$ \\
\hline 4 & Plating frequency & Plfr & Number of plating operations & $0-4$ \\
\hline 5 & Number of operations & Noo & $\begin{array}{c}\text { Number of operations to produce the } \\
\text { order }\end{array}$ & $16-71$ \\
\hline 6 & Number of Prepregs & $N P P$ & Number of Prepregs for lamination & $1-50$ \\
\hline 7 & Scrap units in a set & Sus & Allowed maximum scrap units in a set & $0-8$ \\
\hline 8 & Photoelectric board & Photb & \multirow{3}{*}{$\begin{array}{c}\text { Whether the order is the specified } \\
\text { board }\end{array}$} & \multirow{7}{*}{$0 / 1$} \\
\hline 9 & High frequency board & Highfb & & \\
\hline 10 & Test board & Semictb & & \\
\hline 11 & Negative film plating & $N f l p$ & $\begin{array}{l}\text { Whether the order takes negative film } \\
\text { plating }\end{array}$ & \\
\hline 12 & Tinning copper & Tinc & Whether the order has tinning copper & \\
\hline 13 & IPCIII standard & IPCIII & Whether the order takes IPCIII or & \\
\hline 14 & Huawei standard & Huawei & Huawei standard & \\
\hline \multicolumn{5}{|c|}{ Feature of internal/outer layer line } \\
\hline 15 & $\begin{array}{c}\text { Minimum line width in internal } \\
\text { layer (mil) }\end{array}$ & Mwil & $\begin{array}{l}\text { Minimum line width or space in core } \\
\text { boards }\end{array}$ & $0.1-100$ \\
\hline 16 & $\begin{array}{c}\text { Minimum line space in internal } \\
\text { layer (mil) }\end{array}$ & Mlsil & \multirow{3}{*}{$\begin{array}{c}\text { Minimum line width or space in outer } \\
\text { layer }\end{array}$} & $0.1-137.66$ \\
\hline 17 & $\begin{array}{c}\text { Minimum line width in outer } \\
\text { layer (mil) }\end{array}$ & Mwol & & $1-157.5$ \\
\hline 18 & $\begin{array}{c}\text { Minimum line space in outer } \\
\text { layer (mil) }\end{array}$ & Mlsol & & $1.2-290$ \\
\hline 19 & Average residual rate & Arcr & Average residual rate of copper layer & $0.15 \%-94.75 \%$ \\
\hline \multicolumn{5}{|c|}{ Feature and operation information of hole } \\
\hline 20 & Solder resist plug hole & Srph & \multirow{4}{*}{$\begin{array}{l}\text { Whether the order has the specified } \\
\text { hole related operation }\end{array}$} & \multirow{4}{*}{$0 / 1$} \\
\hline 21 & Plug hole with resin & Phwr & & \\
\hline 22 & Second drilling & Secd & & \\
\hline 23 & Back drilling & $B c d r$ & & \\
\hline \multicolumn{5}{|c|}{ Operation information of character/solder mask } \\
\hline 24 & Character print & Chaprt & \multirow{4}{*}{$\begin{array}{c}\text { Whether the order has the specified } \\
\text { character/solder mask related } \\
\text { operation }\end{array}$} & \multirow{4}{*}{$0 / 1$} \\
\hline 25 & White oil solder mask & White & & \\
\hline 26 & Blue oil solder mask & Blue & & \\
\hline 27 & Black oil solder mask & Black & & \\
\hline \multicolumn{5}{|c|}{ Surface finishing operation options } \\
\hline 28 & Hot air solder leveling & Hasl & \multirow{8}{*}{$\begin{array}{l}\text { Whether the order takes the specified } \\
\text { surface finishing operation }\end{array}$} & \multirow{8}{*}{$0 / 1$} \\
\hline 29 & Lead-free hot air solder leveling & Lfhasl & & \\
\hline 30 & Entek & Osp & & \\
\hline 31 & $\mathrm{Cu} / \mathrm{Ni} / \mathrm{Au}$ pattern plating & Cnapp & & \\
\hline 32 & Gold finger plating & Gfig & & \\
\hline 33 & Gold plating & Godp & & \\
\hline 34 & Soft Ni/Au plating & Snap & & \\
\hline 35 & Immersion $\mathrm{Ag} / \mathrm{Sn} / \mathrm{Au}$ & Iasa & & \\
\hline \multicolumn{5}{|c|}{ Statistic items } \\
\hline 36 & Delivery unit in a panel & Duap & Number of delivery units in a panel & $1-262$ \\
\hline
\end{tabular}


TABLE 1: Continued.

\begin{tabular}{|c|c|c|c|c|}
\hline Number & Variable name & Symbol & Description & Value range \\
\hline 37 & Supplemental feeding frequency & Supff & Material feeding frequency minus 1 & $0-14$ \\
\hline 38 & Required quantity & Reqq & $\begin{array}{l}\text { Demand quantity of delivery unit } \\
\text { minus delivery unit in inventory for } \\
\text { the same order number }\end{array}$ & $1-3,000$ \\
\hline 39 & Required panel & $\operatorname{Reqp}$ & $\begin{array}{c}\text { Reqq/Duap rounded up to the nearest } \\
\text { integer }\end{array}$ & $1-225$ \\
\hline 40 & Feeding quantity & Fedq & Feeding number of delivery units & $2-6296$ \\
\hline 41 & Least feeding panel & $L f p$ & $\begin{array}{c}\text { Reqq/(1 - scrap rate }) \text { rounded up to } \\
\text { the nearest integer }\end{array}$ & $1-245$ \\
\hline 42 & Feeding panel & Fedp & Number of feeding panels & $1-308$ \\
\hline 43 & Scrap quantity & Scraq & Scrap number of delivery units & $0-712$ \\
\hline 44 & Qualified quantity & Qualq & Qualified number of delivery units & $1-6,226$ \\
\hline 45 & Surplus quantity & Surpq & Qualq-Fedq & $0-3,226$ \\
\hline 46 & Delivery unit area $\left(\mathrm{m}^{2}\right)$ & Dunita & Area of a delivery unit & $0.001-0.393$ \\
\hline 47 & Required area $\left(\mathrm{m}^{2}\right)$ & Reqa & Reqq $\times$ Dunita & $0.001-25.74$ \\
\hline 48 & Feeding area $\left(\mathrm{m}^{2}\right)$ & Feda & Fedq $\times$ Dunita & $0.011-42.63$ \\
\hline 49 & Scrap area $\left(\mathrm{m}^{2}\right)$ & Scraa & Scraq $\times$ Dunita & $0-15.39$ \\
\hline 50 & Qualified area $\left(\mathrm{m}^{2}\right)$ & Quala & Qualq $\times$ Dunita & $0.009-37.49$ \\
\hline 51 & Surplus area $\left(\mathrm{m}^{2}\right)$ & Surpa & Surpq $\times$ Dunita & $0-25.45$ \\
\hline 52 & Supplemental feeding rate & Supfr & $\begin{array}{c}\text { Supff in a certain period/number of } \\
\text { orders } \times 100 \%\end{array}$ & $19.84 \%$ \\
\hline 53 & Scrap rate & Scrar & Scraa/Feda $\times 100 \%$ & $0 \%-72.58 \%$ \\
\hline 54 & Qualified rate & Qualr & Quala/Feda $\times 100 \%$ & $27.42 \%-100 \%$ \\
\hline 55 & Surplus rate & Surpr & Surpa/Reqa $\times 100 \%$ & $0-827.18 \%$ \\
\hline 56 & Historical qualified rate & Hquar & $\begin{array}{l}\text { The Qualr for the same order number } \\
\text { in the past } 2 \text { years }\end{array}$ & $8.824 \%-100 \%$ \\
\hline
\end{tabular}

Note. New orders having no Hquar are replaced by the Qualr for orders having the same layer number and surface finishing operation during the past 2 years.

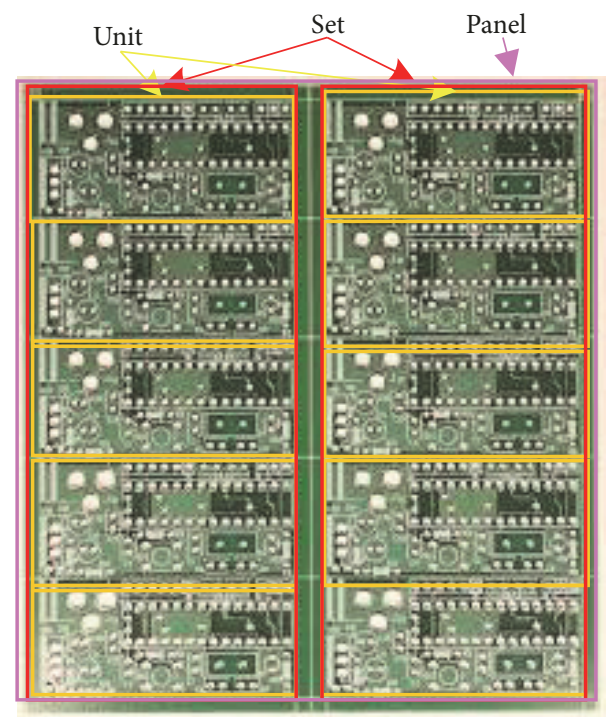

FIGURE 1: Structure of a PCB panel.

or unit before delivery depending on the requirement of customers. The relation between set, unit, and panel specified in Table 1 is illustrated in Figure 1, in which each panel consists of 10 units in the PCB order. Suppose the customer's required quantity and required panel of the PCB order given in Figure 1 are 90 units and 9 panels, respectively. If the initial feeding is 100 units (10 panels) but finally ended up with 95 qualified units due to scrap rate (i.e., $(100-95) \times$ Dunita $/ 100 \times$ Dunita $\times 100 \%=5 \%$ in this example) after production, then the surplus quantity is 5 units $(=95-90)$ and therefore feeding 10 panels is more reasonable to reduce the redundancy of the customized orders. Conversely, it will result in supplemental feeding if we feed only 9 panels initially due to the scrap rate.

On this basis, 30,117 samples of the orders placed between October 31, 2015, and October 31, 2016, were exported with careful audit for erroneous and missing values. The number of the orders for each required panel is illustrated in Figure 2. It can be seen that the required panel is less than 30 in most of the case, which represents a typical customer-oriented small batch template production in PCB industry.

\section{Methodology}

The main flow of the proposed approach (MSCM-ANN) is presented in Figure 3, and various aspects of MSCM-ANN are discussed in detail in the following subsections. The multivariate boxplot, MSCM-based partition, and neighborhood 


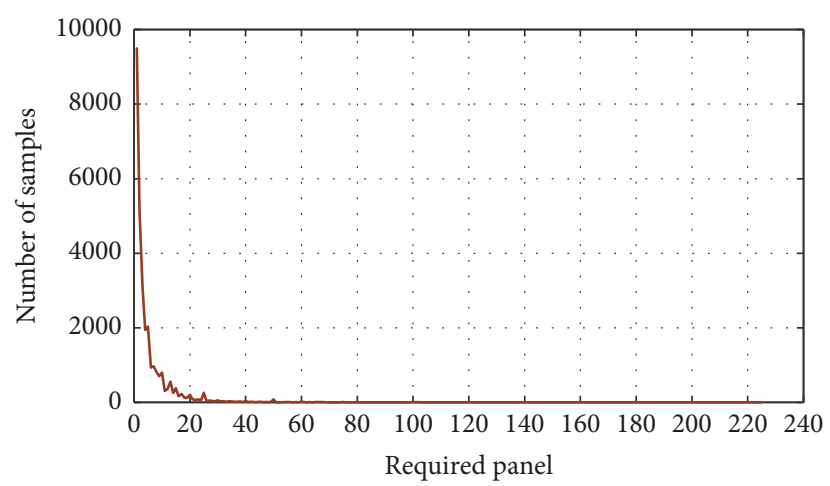

FIGURE 2: Number of orders for each required panel.

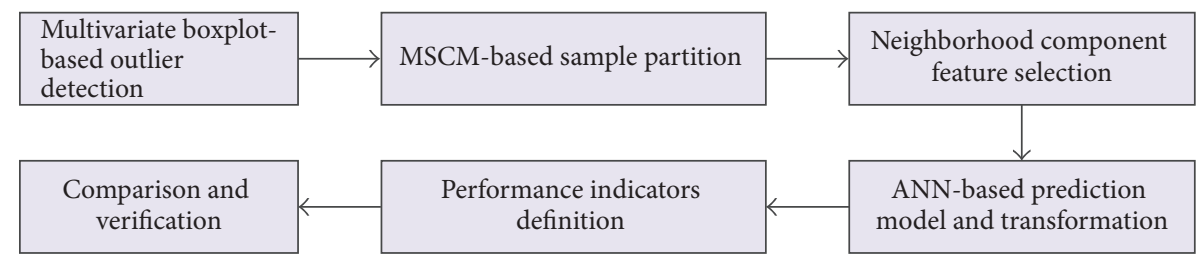

FIGURE 3: Main flow of the proposed approach.

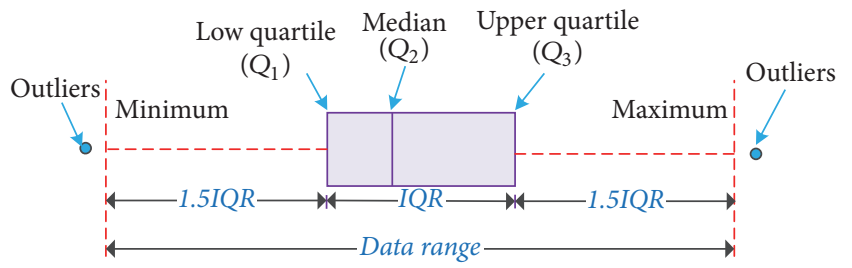

FIgURE 4: Description of boxplot.

component feature selection are the enabling technique of DM considering structural change influence; and the ANNbased prediction model is the mining technique to predict scrap rate; meanwhile, the transformation of scrap rate to surplus rate and supplemental feeding rate is conducted. Performances of the proposed MSCM-ANN will be compared to the ANN and manual feeding based on the same 29,157 samples after outlier detection of original 30,117 samples. Some statistic results of manual feeding are given in Table 1 by the variables 40 to 56. MSCM-ANN and ANN were implemented in Matlab $^{\circledR}$ version 2017a.

3.1. Multivariate Boxplot-Based Outlier Detection. Identification of outliers and the consequent removal are a part of the data screening process which should be done routinely before analyses $[23,24]$. There are various methods of outlier detection. Some are graphical such as normal probability plot, and others are model-based approaches which assume that the data are from a normal distribution [24]. Boxplot is a hybrid of the above two mechanisms for exploring both symmetric and skewed quantitative data, and it can also identify infrequent values from categorical data. Figure 4 shows a description of boxplot, and one could define an outlier as any observation outside the range $\left[Q_{1}-1.5 \mathrm{IQR}, Q_{1}+\right.$ 1.5IQR], where IQR $=Q_{2}-Q_{1}$ and is the interquartile range which contains $50 \%$ of the data. The value is a lower outlier, if $x<Q_{1}-1.5 \mathrm{IQR}$, and an upper outlier, if $x>Q_{1}-1.5 \mathrm{IQR}$.

Detection of outlier sample according to scrap rate, as the target variable of the prediction here, can reduce the impact of accidents that may be caused by machine break, wrong operation, and so on. However, scrap rate related outlier detection influenced by multivariable with structural change does not guarantee they are subject to normal distribution. The modification of the boxplot, called multivariate boxplot here, is developed to identify and discard outliers. The main procedure can be described in Algorithm 1.

3.2. Multiple Structural Change Model-Based Sample Partition. The required panel has significant influence on the scrap rate according to expert experience and initial analysis. The average scrap rate of the orders with different required panel is illustrated in Figure 5. The curve shows declining tendency when the required panel is less than 9 but presents great fluctuations when the required panel is larger than 30 . The multiple structural change of average scrap rate versus the required panel may require separate features and prediction models to improve the prediction accuracy.

MSCM was employed to explore multiple structural changes of samples and partition samples. MSCM was initially developed by Bai and Perron $[25,26]$ to address problem of online (time serial related) multiple linear regression (MLR) with multiple structural change along with time. MSCM takes least squares method to detect the number of break points and estimate the change position. Here, the required panel in ascending sort order is considered as time serial related date, and the scrap rate is taken as the online 
Input: $N$ samples; categorical variable set $f_{\text {set }}=\left\{f_{1}, f_{2}, \ldots, f_{u}\right\}$

Output: Outlier frequency for each sample $f q=\left\{f q_{1}, \ldots, f q_{N}\right\}$

Initialize: $f q=\left\{f q_{1}, \ldots, f q_{N}\right\}=\{0,0, \ldots, 0\}$

for $i=1: u$ do

set $V a_{i}=\left\{v_{i 1}, v_{i 2}, \ldots, v_{i s}\right\}$ as the values of feature $f_{i}$

Plot a boxplot of scrap rate for each values in $V a_{i}$, and detect samples with scrap rate out of

$\left[Q_{1}-1.5 \mathrm{IQR}, Q_{1}+1.5 \mathrm{IQR}\right]$ for each boxplot; and outlier frequency of these samples are added by 1 .

End for

Remove the samples corresponding to $f q$ with $f q_{k} \geq 2,1 \leq k \leq N$. Return $f q=\left\{f q_{1}, \ldots, f q_{N}\right\}$

Algorithm 1: Multivariate boxplot-based outlier detection of scrap rate.

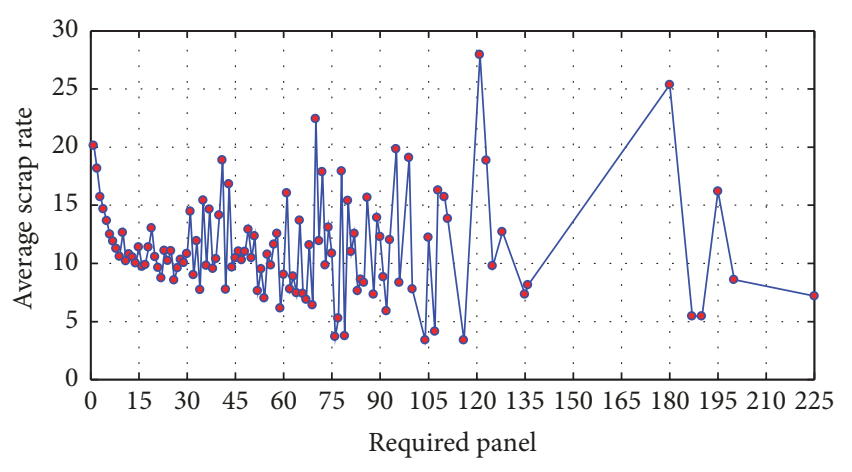

FIGURE 5: Average scrap rates with different required panel.

regression objective. Then the online MLR of the scrap rate with $m$ breaks ( $m+1$ regimes) can be expressed as scrapr ${ }_{t}=$ $z_{t}^{\prime} \delta_{j}+u_{t}, t=N_{j-1}+1, \ldots, N_{j}$, for $j=1, \ldots, m+1$ with the convention $N_{0}=0$ and $N_{m+1}=N$. In this model, $\operatorname{scrpr}_{t}$ is the observed scrap rate, and $z_{t}$ is vectors of independent variable, but only the layer number, required panel, and number of operations are considered here. $\delta_{j}(j=$ $1, \ldots, m+1)$ is the corresponding vectors of coefficients, and $u_{t}$ is the disturbance. The break points, $N_{1}, \ldots, N_{m}$, are explicitly treated as unknowns. The purpose is to estimate the unknown coefficients together with the break points when $N$ observations (samples) on $\operatorname{scrapr}_{t}, z_{t}$ are available.

For each $m$-partition $\left(N_{1}, \ldots, N_{m}\right),\left(\widehat{\delta}_{1}, \ldots, \widehat{\delta}_{j}\right)$ as the estimated results of $\delta_{j}$ are obtained by minimizing sum of squared residuals $S=\sum_{i=1}^{m+1} \sum_{t=N_{i-1}+1}^{N_{i}}\left[\text { scrapr }_{t}-z_{t}^{\prime} \delta_{i}\right]^{2}$. Substituting them in the objective function and denoting the resulting as $S_{N}\left(N_{1}, \ldots, N_{m}\right)$, the estimated partition to determine the break points and related change position are such that $\left(\widehat{N}_{1}, \ldots, \widehat{N}_{m}\right)=\arg \min _{N_{1}, \ldots, N_{m}} S_{N}\left(N_{1}, \ldots, N_{m}\right)$ [26].

Significance test for the structural changes based on newly introduced statistic items $\sup F(l), U D_{\max }, W D_{\max }$, and $\sup F_{T}(l+1 / l)$ is conducted. The $\sup F(l)$ is the $F$ test with original hypothesis $m=0$ and alternative hypothesis $m=l$. Then $U D_{\max }=\max _{1 \leq l \leq L} \sup F_{T}(l)$ and $W D_{\max }=$ $\max _{1 \leq l \leq L} w_{l} \sup F_{T}(l)$ are introduced to check whether there is the structural change in the model, in which $w_{l}$ is set as the weight of $p$ value based on $\sup F_{T}(l)$ hypothesis test, and $L$ is the maximum number of $l$. The number of the break points for the model is determined according to $\sup F_{T}(l+1 / l)(l \geq$ $1)$, and $F_{T}(l+1 / l)$ is the $F$ test with original hypothesis having $l$ break points and alternative hypothesis having $l+1$ break points. Therefore the samples can be partitioned into subgroups according to the break points and corresponding change position $[25,26]$. All the estimation and hypothesis tests are conducted based on Matlab code provided by $\mathrm{Qu}$ [27].

3.3. Neighborhood Component Feature Selection. It is necessary to employ some feature selection methods to remove irrelevant and redundant features to reduce the complexity of analysis and the generated models and also improve the efficiency of the whole modeling processes [28-30]. Wrappers, embedded, and filter are three types of the approaches developed for feature selection [31]. In this study, neighborhood component feature selection (NCFS) was applied for each group of the samples. NCFS is an embedded method for feature selection with regularization to learn feature weights for minimization of an objective function that measures the average leave-one-out regression loss over the training data [32].

Given $n$ observations, $S=\left\{\left(x_{i}, y_{i}\right), i=1,2, \ldots, n\right\}$, where $x_{i} \in R^{p}$ are the feature vectors and $y_{i} \in R$ are response (scrap rate). In this study, the aim is to predict the response given the training set $S$. Consider a randomized regression model that randomly picks a point $(\operatorname{Ref}(x))$ from $S$ as the "reference point" for $x$ and sets the response value at $x$ equal to the response value of the reference point $\operatorname{Ref}(x)$. Now consider the leave-one-out application of this randomized regression model, that is, predicting the response for $x_{i}$ using the data in $S^{-i}=S /\left(x_{i}, y_{i}\right)$. The probability that point $x_{j}$ is picked as the reference point for $x_{i}$ is

$$
p_{i j}=P\left(\operatorname{Ref}(x)=x_{j} \mid S^{-i}\right)=\frac{k\left(d_{w}\left(x_{i}, x_{j}\right)\right)}{\sum_{j=1, j \neq i}^{N} k\left(d_{w}\left(x_{i}, x_{j}\right)\right)},
$$

where $d_{w}\left(x_{i}, x_{j}\right)=\sum_{r=1}^{m} w_{r}^{2}\left|x_{i r}-x_{j r}\right|, w_{r}, r=1,2, \ldots, m$, are the feature weights, and $k$ is the kernel function. Suppose $k(z)=\exp (-z / \sigma)$ as suggested in [32], where $\sigma$ is set to 1 after standardizing the dependent value to have zero mean and unit standard deviation.

Let $\widehat{y}_{i}$ be the response value of the randomized regression model and let $y_{i}$ be the actual response for $x_{i}$. And let $l$ be a 


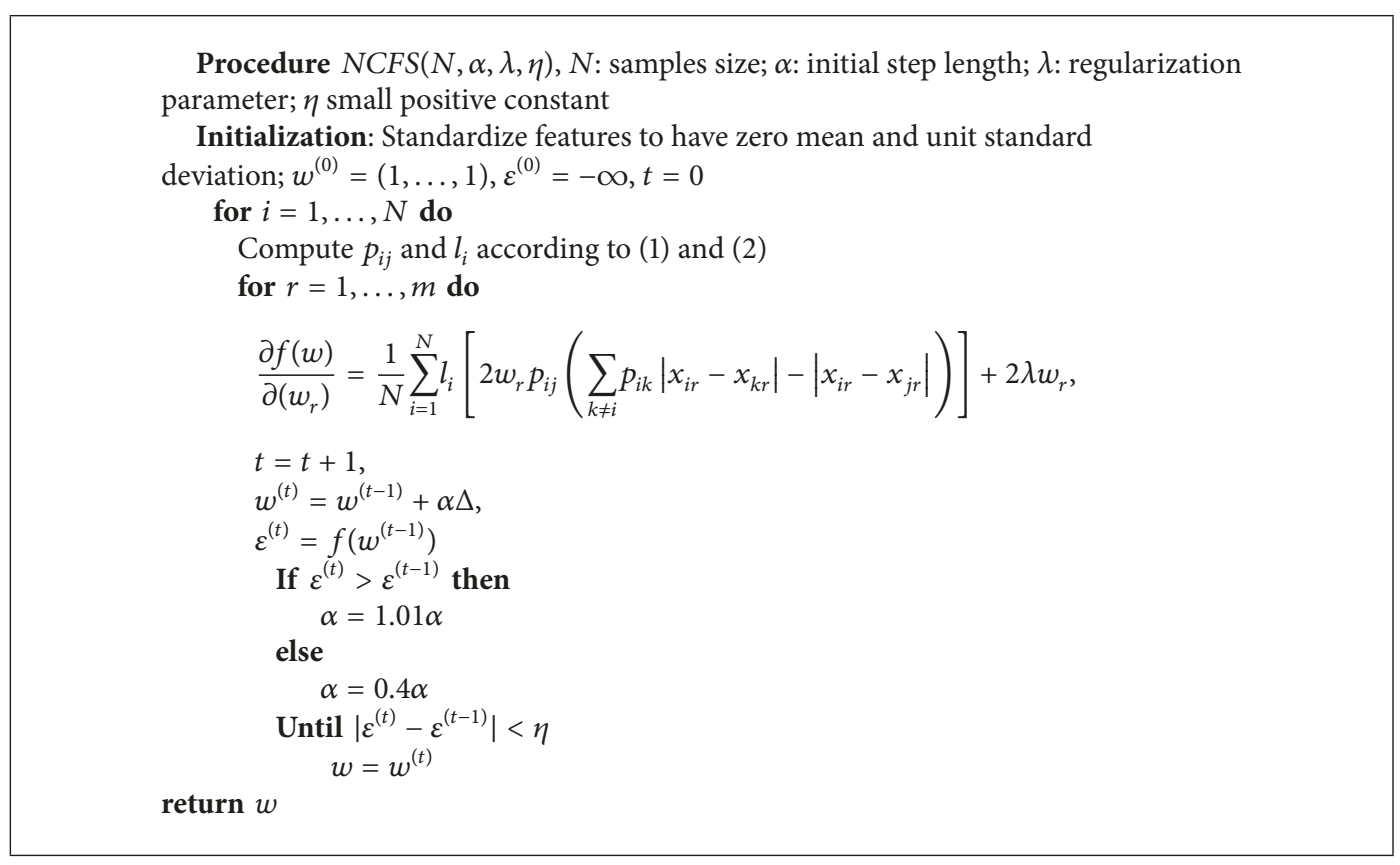

Algorithm 2: Neighborhood component feature selection.

loss function that measures the disagreement between $\widehat{y}_{i}$ and $y_{i}$. Then, the average value of $l\left(y_{i}, \widehat{y}_{i}\right)$ is

$$
l_{i}=E\left(l\left(y_{i}, \widehat{y}_{i}\right) \mid S^{-i}\right)=\sum_{j=1, j \neq i}^{N} p_{i j} \times l\left(y_{i}, y_{j}\right) \text {. }
$$

After adding the regularization term $\lambda \sum_{r=1}^{m} w_{r}^{2}$, the objective function for minimization is

$$
\begin{aligned}
f(w) & =\frac{1}{N} \sum_{i=1}^{N} l_{i}+\lambda \sum_{r=1}^{m} w_{r}^{2} \\
& =\frac{1}{N} \sum_{i=1}^{N} \sum_{j=1, j \neq i}^{N} p_{i j} \times l\left(y_{i}, y_{j}\right)+\lambda \sum_{r=1}^{m} w_{r}^{2} .
\end{aligned}
$$

The loss function for $l\left(y_{i}, y_{j}\right)$ here is the mean absolute deviation defined as $\sum_{i=1}^{N}\left|y_{i-} y_{j}\right| / N$. The main procedure of NCFS for regression feature selection can be summarized in Algorithm 2.

\subsection{Neural Network-Based Prediction Model and Transforma-} tion. The most frequently used DM method for prediction is ANN. An ANN is network neurons that consist of propagation function and activation function, which receives the input, changes their internal state (activation) according to the input, and produces the output depending on the input and activation [33]. Despite the black box mechanism of ANN, it has been widely used in prediction problems demonstrating reasonable results as scrutinized in the literature [34]. ANN with their successful experience in forecasting diverse problems are among the most accurate and trustworthy used models. Their ability to learn from incomplete datasets in order to predict the unseen section of data besides their capability of modeling the problem with the least available data and estimating almost all continuous functions has made them attractive enough to be used in prediction problems [34]. Köksal et al. [5] reviewed the reported performance of the DM methods and also pointed out that the ANN performance is mostly compared to the performance of the classical statistical modeling method such as multiple linear regressions (MLR), and better performance of ANN can naturally be observed in multidimensional data since these are powerful tools in modeling nonlinear relationships.

In this study, three-layer back propagation ANN was taken to predict the scrap rate; then it was transformed to determine the predicted surplus rate and supplemental feeding rate, two most concerned performances for material feeding optimization. The architecture of ANN is set by trial and error, and the number of nodes in hidden layer is set to $\max \left(3,\left(n_{i}+1\right) / 20\right)$, in which $n_{i}$ is the number of input features. The ANN-based architecture for the scrap rate prediction and material feeding optimization is illustrated in Figure 6, in which a neuron (in the hidden layer or the output layer) $j$ receives the outputs $a_{1}, a_{2}, \ldots, a_{n^{[l]}}$ of other neurons $1, \ldots, n^{[l]}$ which are connected to $j$ with bias $\theta_{j}$, and the propagation function of neuron $j$ is defined as $z_{j}^{[l]}=$ $\sum_{i=1}^{n^{[l-1]}} w_{i j} a_{i}+\theta_{j}$, in which the superscript $[l]$ denotes the $l$ th layer and $n^{[l-1]}$ is the number of units of the $(l-1)$ th layer. The results of propagation function are further processed by sigmoid activation function; that is, $a_{j}=f\left(z_{j}\right)=1 /\left(1+e^{-z_{j}}\right)$.

The transformation can be conducted according to (4)-(5) following the hypothesis that each feeding panel of an order has the same scrap probability, and scrap rate for an order will not change along with the predicted feeding area. Therefore, 


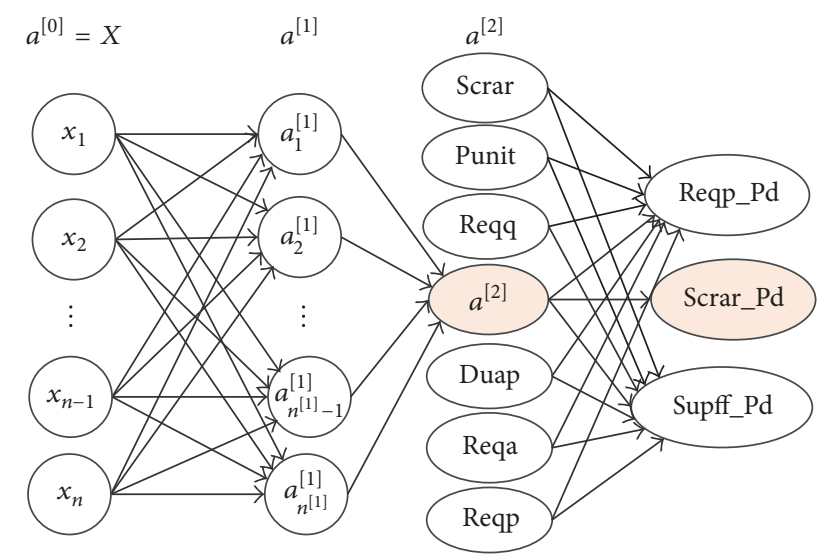

FIGURE 6: ANN-based architecture for the prediction of scrap rate and material feeding optimization (one can refer to Tables 1 and 2 for the notations).

TABLE 2: Prediction results related variables.

\begin{tabular}{lc}
\hline Variable & Symbol \\
\hline Predicted scrap rate & Scrar_Pd \\
Predicted feeding quantity & Fedq_Pd \\
Predicted feeding panel & $F e d p \_P d$ \\
Predicted feeding area & Feda_Pd \\
Predicted scrap area & Scraa_Pd \\
Predicted surplus rate & Surpr_Pd \\
Predicted supplemental feeding frequency for an order & Supff_Pd \\
Predicted supplemental feeding rate & Supfr_Pd \\
\hline
\end{tabular}

the predicted surplus rate and supplemental feeding rate can be calculated according to (6)-(8), and related variables are presented in Table 2.

The predicted feeding quantity and panel for each order are described as

$$
\begin{aligned}
& \text { Fedq_Pd }=\frac{\text { Reqq } /\left(100-S_{\text {Scrar_Pd }} P\right.}{100}, \\
& \text { Fedp_Pd }=\left\lceil\frac{\text { Fedq_Pd }}{\text { Duap }}\right\rceil,
\end{aligned}
$$

in which Duap is the delivery unit in a panel given in Table 1. Then the predicted feeding quantity, area, and scrap area should be revised accordingly:

$$
\begin{aligned}
& \text { Fedq_Pd }=\text { Fedp_Pd } \times \text { Duap, } \\
& \text { Feda_Pd }=\text { Fedq_Pd } \times \text { Punita, } \\
& \text { Scraa_Pd }=\text { Feda_Pd } \times \text { Scrar },
\end{aligned}
$$

Thus the predicted surplus rate of each order can be defined as

$$
\text { Surp_Pd }=\frac{\left(\text { Feda } P d-\text { Reqa }-S c r a a \_P d\right)}{\text { Reqa }} \times 100 \% .
$$

The predicted supplemental feeding frequency for each order is specified as

$$
\text { Supff_Pd }= \begin{cases}1, & \text { Feda_Pd }- \text { Reqa }- \text { Scraa } P d<0 \\ 0, & \text { otherwise. }\end{cases}
$$

Reqa is the required area defined in Table 1 . Therefore, the predicted surplus rate for these orders with $\operatorname{Supff} P d_{i}=0$ can be calculated by

$$
\text { Surpr_Pd }=\frac{\sum_{i=1}^{N^{\prime}}\left(\text { Feda }_{-} P d_{i}-\text { Reqa }_{i}-S c r a a_{-} P d_{i}\right)}{\sum_{i=1}^{N^{\prime}} \operatorname{Reqa}_{i}} .
$$

$N^{\prime}$ is the number of samples with Supff $P d_{i}=0,1 \leq$ $i \leq N$. The Surpr $P d$ for these orders with Supff_Pd $d_{i}=1$ is not considered here because the surplus area cannot be determined before the supplemental feeding is finished. But their surplus rate is always lower than the Surpr_Pd defined in (10) in practice.

The supplemental feeding rate for all the samples can be defined as

$$
\text { Supfr } P d=\frac{\sum_{i=1}^{N} \operatorname{Supff} \_P d_{i}}{N} \times 100 \% .
$$

The surplus rate and supplemental feeding rate of the manual feeding can be computed by

$$
\begin{aligned}
& \text { Surpr }=\frac{\sum_{i=1}^{N}\left(\text { Feda }_{i}-\text { Reqa }_{i}-\text { Scraa }_{i}\right)}{\sum_{i=1}^{N} \text { Reqa }_{i}}, \\
& \text { Supfr }=\frac{\sum_{i=1}^{N} \text { Supff }_{i}}{N} \times 100 \% .
\end{aligned}
$$

3.5. Performance Indicators. In order to evaluate the effectiveness of the model, the following evaluation indicators are used [35]. The mean squared error (MSE) is the average of square sums between predicted data $\widehat{y}_{i}$ and original data $y_{i}$, which can be described as

$$
\text { MSE }=\frac{\sum_{i=1}^{N}\left(\hat{y}_{i}-y_{i}\right)^{2}}{N} .
$$

The mean absolute error (MAE) is the average of the sum of the absolute difference between observed values and estimated values. It can be expressed as

$$
\mathrm{MAE}=\frac{\sum_{i=1}^{N}\left|\hat{y}_{i}-y_{i}\right|}{N} .
$$

The mean absolute percentage error (MAPE) is the average of the sum of the normalized absolute difference between observed values and estimated values. The formula is written as

$$
\operatorname{MAPE}=\frac{1}{N} \sum_{i=1}^{N}\left|\frac{\hat{y}_{i}-y_{i}}{y_{i}}\right| \times 100
$$




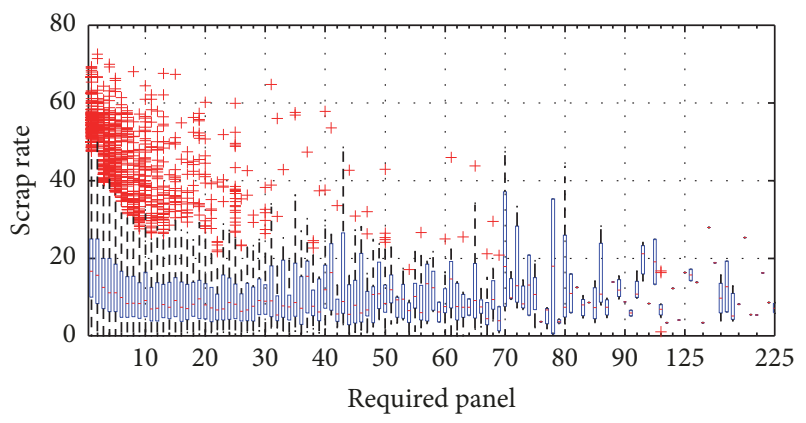

(a)

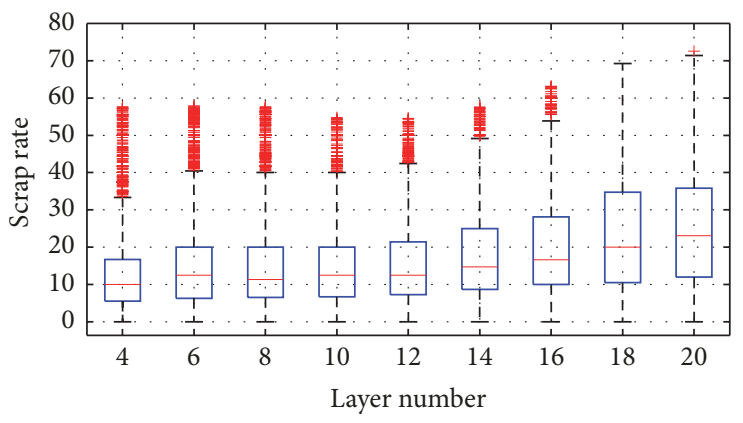

(b)

FIgURE 7: (a) Boxplot for different value of required panel. (b) Boxplot for different value of layer number.

TABLE 3: Significance test of break points.

\begin{tabular}{lcc}
\hline Test variables & Test value & Critical value [18] \\
\hline$U D_{\max }$ & $8,543.4119^{*}$ & 10.17 \\
$W D_{\max }$ & $8,543.4119^{*}$ & 10.91 \\
$\sup F_{T}(2 \mid 1)$ & $4,030.0921^{*}$ & 11.14 \\
$\sup F_{T}(3 \mid 2)$ & $3,297.9988^{*}$ & 12.16 \\
$\sup F_{T}(4 \mid 3)$ & $1,437.4137^{*}$ & 12.83 \\
$\sup F_{T}(5 \mid 4)$ & 793.6005 & 13.45
\end{tabular}

5 break points corresponding to the required panel intervals $1,2,3$, $4-6,7-19$, and greater than 19

${ }^{*}$ A statistic significance at the $5 \%$ level.

where $N$ is the number of samples. The final purpose is to determine the feeding panel for each order on the basis of the predicted scrap rate, and $y_{i}$ and $\hat{y}_{i}$ are replaced by the least feeding panel and predicted feeding panel, respectively. Then the deviation of the predicted feeding and the manual feeding can be computed according to $\widehat{y}_{i}-y_{i}$ and Fed $p_{i}-y_{i}$ for sample $i, 1 \leq i \leq N$, respectively. The error diagram can be drawn as a distribution of the deviation for all samples. Combined with the aforementioned predicted surplus rate and supplemental feeding rate for material feeding optimization, the final performance will be evaluated by the five indicators.

\section{Results and Discussion}

According to the multivariate boxplot approach described in Section 3.1, 960 outliers were trimmed and 29,157 samples left. Figure 7 shows the boxplot of the scrap rate for different value of the required panel and the layer number. Figure 7 illustrates that the outliers are shifted by the values of the required panel and the layer number, and therefore outlier detection considering different feature values is more reasonable.

Significance test for break points of the samples according to $U D_{\max }, W D_{\max }$, and $\sup F_{T}(l+1 / l)$ was conducted based on default parameters given in [27], and the results are given in Table 3. The values of $U D_{\max }$ and $W D_{\max }$ indicated that the samples have significant structural change at $5 \%$ level, and the values of $\sup F_{T}(l+1 / l)$ showed that 5 break points are significant. The final break position of ascending sorted samples according to the required panel was 8,935 , $13,995,17,003,21,791$, and 27,491 . Therefore the samples were partitioned into 6 groups with indexes $1-6$ which corresponds to the samples with the required panels $1,2,3,4-6,7-19$, and greater than 19, respectively. The samples in group 6 can still be segmented for $\sup F_{T}(5 \mid 4)$ greater than the critical value at $5 \%$ level [25]. However, the sample size in group 6 was small (1666 samples) and the average of scrap rate greatly fluctuated which can be seen from Figures 1 and 6(a). Thus further partition for the samples in group 6 was not conducted.

Then NCFS was conducted for each group of the samples in which the initial step length was set to 0.9 and small positive constant $\eta$ was set to $10^{-4}$. 5 -fold cross-validation instead of a single test was conducted to optimize regularization parameter $\lambda$ initialized with 20 randomly selected values between $0-1.2 \times 10^{-3}$ according to [32], and the $\lambda$ value that minimizes the mean loss across the cross-validation was selected to fit NCFS. Figure 8(a) shows that the loss performance for 20 different $\lambda$ values for the group of the samples with the required panels between 7 and 19 and the fourth $\lambda$ corresponding to the lowest mean loss was selected as the regularization parameter for NCFS. Figure 8 (b) illustrates the indexes of the selected features based on the selected $\lambda$. Final selected features for different group of the samples and all samples as a whole are given in Table 4. Difference of the selected features indicates that the samples with the different features may distribute in different regimes. However, layer number, Rogers material, number of operations, Huawei standard, plug hole with resin, second drilling, back drilling, $\mathrm{Cu} / \mathrm{Ni} / \mathrm{Au}$ pattern plating, gold finger plating, gold plating, delivery unit area, and historical qualified rate are critical features for most of the samples, which means that different values of these features will influence the scrap rate greatly in general, and these selected features also match well with the experience of experts from the factory.

The $70 \%, 15 \%$, and $15 \%$ of the mutually exclusive samples were randomly selected as training, validation, and test data for each partitioned group of the samples, and the sample sizes for each group are given in Table 5. Prediction models of MSCM-ANN were trained, validated, and tested for each group of the samples with 5 runs based on the corresponding selected features while the ANN was trained, validated, and 
TABLE 4: Selected features for different group of the samples.

\begin{tabular}{|c|c|c|c|c|c|c|c|c|}
\hline \multirow{2}{*}{ Index } & \multirow{2}{*}{ Feature symbol } & \multicolumn{7}{|c|}{ Sample group } \\
\hline & & 1 & 2 & 3 & 4 & 5 & 6 & All \\
\hline 1 & $P t$ & & & & & & $\nabla$ & $\nabla$ \\
\hline 2 & Ln & $\diamond$ & $\diamond$ & $\diamond$ & $\diamond$ & $\diamond$ & $\diamond$ & 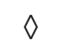 \\
\hline 3 & Ro & & $\diamond$ & $\diamond$ & & $\diamond$ & $\diamond$ & 0 \\
\hline 4 & $P l f r$ & & & & & & $\diamond$ & $\diamond$ \\
\hline 5 & $\mathrm{Noo}$ & & & & $\diamond$ & $\diamond$ & 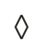 & 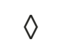 \\
\hline 6 & $N P P$ & & & & & & & $\diamond$ \\
\hline 7 & Sus & & & & & & & \\
\hline 8 & Photb & $\diamond$ & $\Delta$ & & & & & \\
\hline 9 & $H i g h f b$ & $\diamond$ & & & & $\diamond$ & & \\
\hline 10 & Semictb & $\diamond$ & $\diamond$ & $\diamond$ & & & & \\
\hline 11 & $N f l p$ & & & & & & & \\
\hline 12 & Tinc & & & & & $\diamond$ & $\diamond$ & $\diamond$ \\
\hline 13 & IPCIII & & & & & & & \\
\hline 14 & Huawei & & 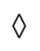 & $\diamond$ & $\diamond$ & $\diamond$ & $\diamond$ & $\diamond$ \\
\hline 15 & Mwil & & & & & & & \\
\hline 16 & Mlsil & & & & & & & \\
\hline 17 & Mwol & & & & & & & \\
\hline 18 & Mlsol & & & & & & & \\
\hline 19 & Srph & & & & & $\Delta$ & & \\
\hline 20 & Phwr & & $\diamond$ & & $\diamond$ & $\diamond$ & & \\
\hline 21 & Secd & $\diamond$ & $\diamond$ & $\diamond$ & $\diamond$ & $\diamond$ & $\diamond$ & $\diamond$ \\
\hline 22 & $B c d r$ & $\diamond$ & & & $\diamond$ & $\diamond$ & $\diamond$ & $\diamond$ \\
\hline 23 & Chaprt & $\Delta$ & & & $\diamond$ & $\diamond$ & $\Delta$ & 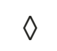 \\
\hline 24 & White & & & & & & $\diamond$ & 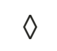 \\
\hline 25 & Blue & & & & & & & \\
\hline 26 & Black & & & & & & & \\
\hline 27 & Chaprt & & & $\diamond$ & & & & \\
\hline 28 & Hasl & & & & & $\diamond$ & $\diamond$ & $\diamond$ \\
\hline 29 & Lfhasl & $\diamond$ & & & & & & \\
\hline 30 & $O s p$ & $\diamond$ & & & & $\diamond$ & & \\
\hline 31 & Cnapp & 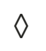 & & $\diamond$ & $\diamond$ & & $\diamond$ & $\diamond$ \\
\hline 32 & Gfig & & & $\diamond$ & $\Delta$ & $\Delta$ & 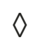 & \\
\hline 33 & Godp & $\diamond$ & 0 & $\diamond$ & $\diamond$ & $\diamond$ & & \\
\hline 34 & Snap & & & & & & & \\
\hline 35 & Iasa & & & & & $\diamond$ & $\diamond$ & $\diamond$ \\
\hline 36 & Reqq & $\diamond$ & & & & & & \\
\hline 37 & Reqpl & & & & & & & $\diamond$ \\
\hline 38 & Dunita & $\diamond$ & $\nabla$ & $\diamond$ & $\diamond$ & $\diamond$ & $\diamond$ & $\diamond$ \\
\hline 39 & Reqa & & & & & & & \\
\hline 40 & Hquar & $\diamond$ & $\Delta$ & $\diamond$ & $\diamond$ & $\diamond$ & $\diamond$ & $\diamond$ \\
\hline
\end{tabular}

Note. Selected features are marked with $\diamond$. The description of features (variables) has been specified in Table 1.

tested from all samples based on the selected features as listed in the last column of Table 4. Comparison of average MSE, MAE, and MAPE of 5 runs for MSCM-ANN, ANN, and the manual feeding is given in Table 6. The results indicate that both MSCM-ANN and ANN have obvious superiority in reducing the three indicators. However, MSCM-ANN can achieve smaller MSE, MAE, and MAPE compared to ANN, which means that the established models considering structural change can further improve the precision.
The average results of each group of the samples achieved by MSCM-ANN, ANN, and manual feeding are given in Table 7. The surplus rate and supplemental feeding rate obtained by the three approaches are given in Table 8 . The following results can be drawn accordingly:

(1) Both MSCM-ANN and ANN can reduce the surplus rate and supplemental feeding rate performances simultaneously compared to the manual feeding as shown in Table 8. MSCM-ANN obtained lower values with $11.96 \%$ 


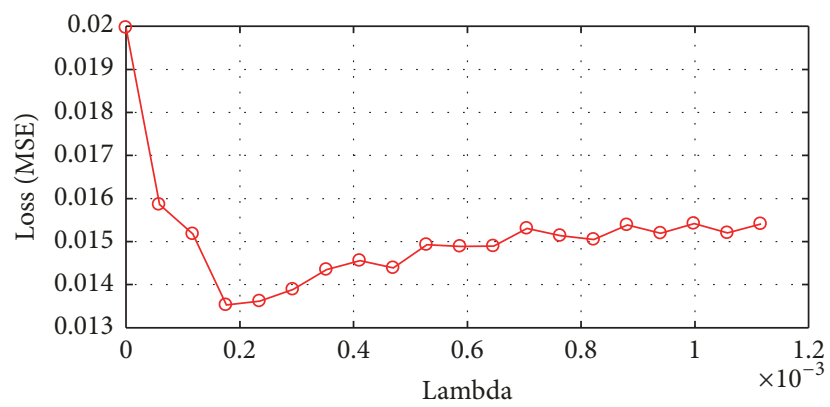

(a)

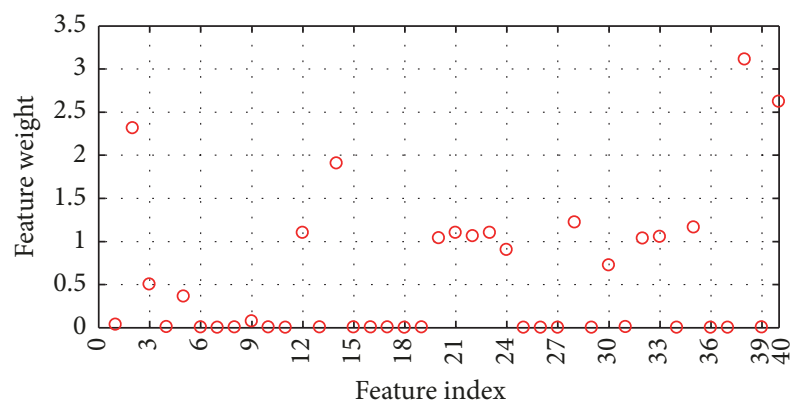

(b)

FIGURE 8: (a) Mean loss performance for 20 different lambda $(\lambda)$ values for samples with required panel between 7 and 19. (b) Feature selection based on NCFS with the lowest loss lambda for samples with required panel between 7 and 19.

TABLE 5: Samples sizes of training, validation, and test data.

\begin{tabular}{|c|c|c|c|c|c|}
\hline Sample group & Panel intervals & Training & Validation & Test & Total number \\
\hline 1 & 1 & 6,255 & 1,340 & 1,340 & 8,935 \\
\hline 2 & 2 & 3,542 & 759 & 759 & 5,060 \\
\hline 3 & 3 & 2,105 & 451 & 451 & 3,007 \\
\hline 4 & $4-6$ & 3,353 & 718 & 718 & 4,789 \\
\hline 5 & $7-19$ & 3,990 & 855 & 855 & 5,700 \\
\hline 6 & $\geq 20$ & 1,166 & 250 & 250 & 1,666 \\
\hline All & All & 20,409 & 4,374 & 4,374 & 29,157 \\
\hline
\end{tabular}

TABLE 6: Performance indicators achieved by different approaches.

\begin{tabular}{|c|c|c|c|c|}
\hline Performance indicators & Samples & MSCM-ANN & ANN & Manual feeding \\
\hline \multirow{4}{*}{ MSE } & Training & 0.719 & 1.803 & 24.272 \\
\hline & Validation & 1.186 & 1.639 & 20.830 \\
\hline & Test & 1.272 & 1.847 & 14.849 \\
\hline & All & 0.872 & 1.786 & 22.342 \\
\hline \multirow{4}{*}{ MAE } & Training & 0.330 & 0.502 & 1.417 \\
\hline & Validation & 0.391 & 0.509 & 1.458 \\
\hline & Test & 0.396 & 0.515 & 1.409 \\
\hline & All & 0.349 & 0.505 & 28.591 \\
\hline \multirow{4}{*}{ MAPE } & Training & 5.402 & 5.784 & 29.088 \\
\hline & Validation & 5.817 & 6.089 & 28.582 \\
\hline & Test & 5.687 & 5.872 & 28.664 \\
\hline & All & 5.522 & 5.783 & 28.591 \\
\hline
\end{tabular}

predicted surplus rate and $11.91 \%$ predicted supplemental feeding rate while ANN achieved $15.16 \%$ and $12.69 \%$ for the two performance indicators, respectively. Better performance of MSCM-ANN may be influenced by more precisely selected features, more reasonable ANN architecture, and well-trained models for each partitioned sample group based on MSCM considering the structural change influence compared to ANN which could not explore the pattern in each partitioned group.

(2) The results in Table 8 indicate that MSCM-ANN and ANN achieved the lower surplus rate but relatively higher supplemental feeding for the samples when the sample group corresponding to the intervals of required panel increases. The main reason is that the required panel that was rounded up to the nearest integer based on the required quantity resulted in high redundancy when the number of the required panels was small, which therefore caused lower supplemental feeding rate. Taking the PCB order in Figure 1, for example, if the required quantity is only 4 units, then it will cause $100 \%((10-2-4) / 4 \times 100 \%)$ surplus rate for feeding one panel with $20 \%$ scrap rate; the supplemental feeding should not be conducted until the scrap rate is greater than $60 \%$. In contrast, lower surplus rate but relatively higher supplemental feeding rate was obtained when the required panel increased with lower surplus, but great fluctuation of the scrap rate may cause insufficient feeding panel and therefore bring about high supplemental feeding frequency.

The predicted scrap rate and predicted supplemental feeding rate on average obtained by MSCM-ANN for the training, validation, and test sample in each group are 


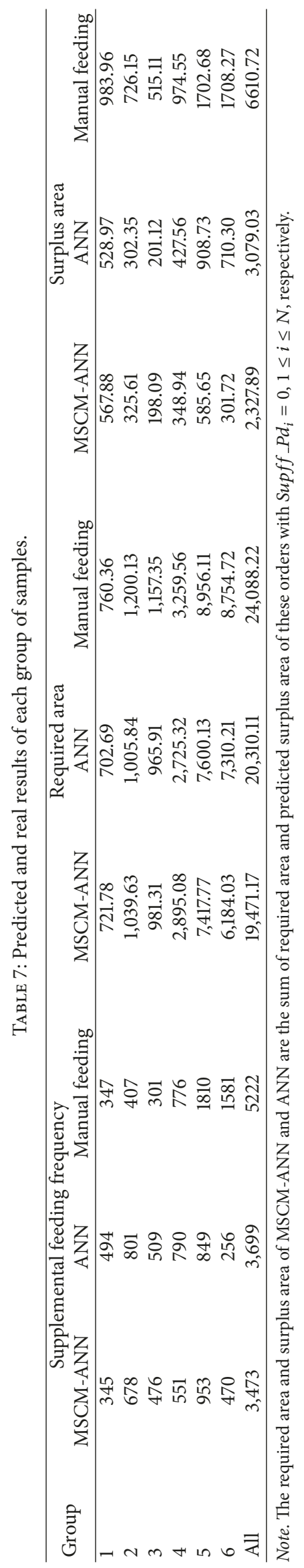


TABLE 8: Comparison of surplus rate and supplemental feeding rate.

\begin{tabular}{|c|c|c|c|c|c|c|}
\hline \multirow{2}{*}{ Group } & \multicolumn{2}{|c|}{ MSCM-ANN } & \multicolumn{2}{|c|}{ ANN } & \multicolumn{2}{|c|}{ Manual feeding } \\
\hline & Surpr_Pd (\%) & Supfr Pd (\%) & Surpr_Pd (\%) & Supfr_Pd (\%) & Surpr (\%) & Supfr (\%) \\
\hline 1 & 78.68 & 3.86 & 75.28 & 5.53 & 129.41 & 3.88 \\
\hline 2 & 31.32 & 13.40 & 30.59 & 15.83 & 60.51 & 8.04 \\
\hline 3 & 20.19 & 15.83 & 20.82 & 16.93 & 44.51 & 10.01 \\
\hline 4 & 12.05 & 11.51 & 15.69 & 16.49 & 29.90 & 16.20 \\
\hline 5 & 7.90 & 16.72 & 11.96 & 14.89 & 14.15 & 31.75 \\
\hline 6 & 4.88 & 28.21 & 9.72 & 15.37 & 19.51 & 94.90 \\
\hline ALL & 11.96 & 11.91 & 15.16 & 12.69 & 27.44 & 17.91 \\
\hline
\end{tabular}

Note. Surpr $P d$ and Supfr $P d$ are the predicted surplus rate and supplemental feeding rate, respectively, and they can be obtained according to the definition specified in Section 3.5 and the data provided in Table 7.

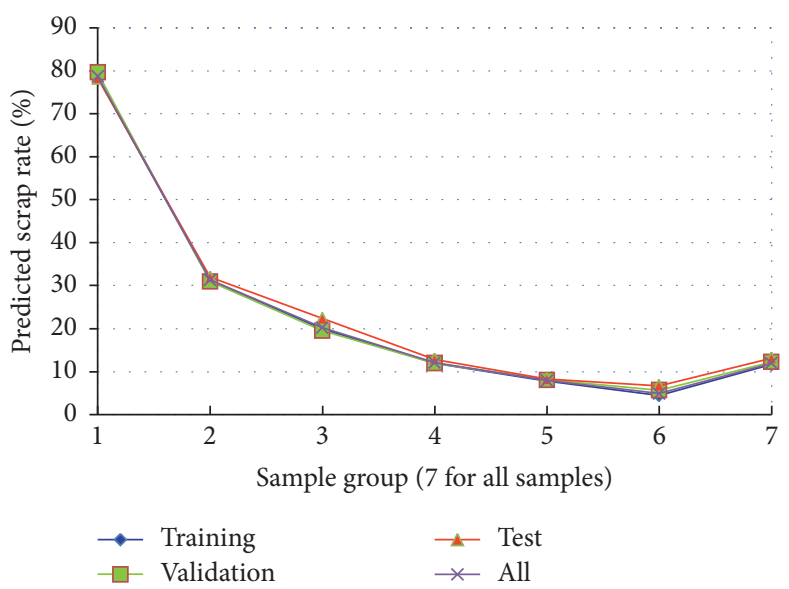

(a)

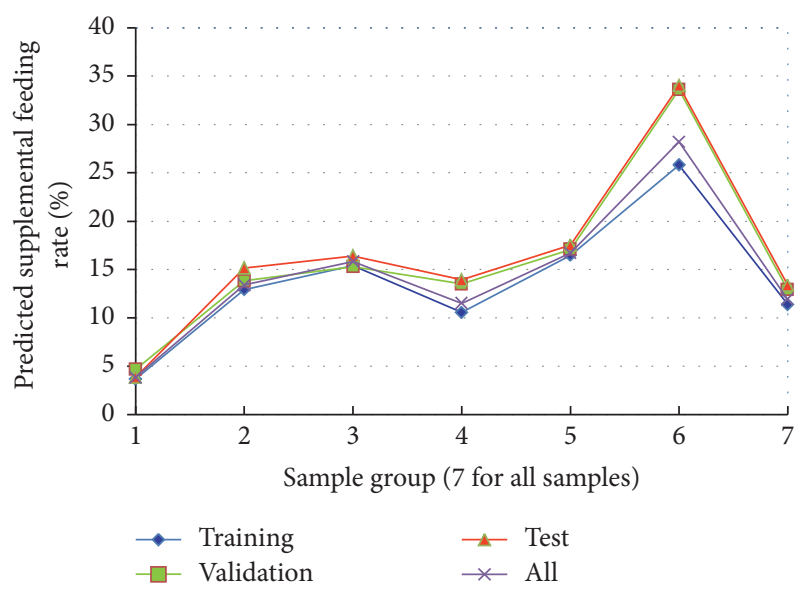

(b)

FIGURE 9: (a) Predicted scrap rate of MSCM-ANN for different samples. (b) Predicted supplemental feeding rate for different samples.

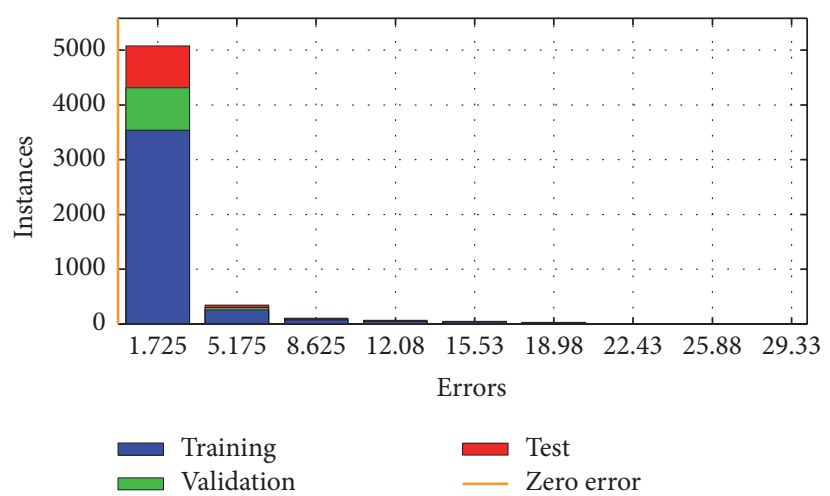

(a)

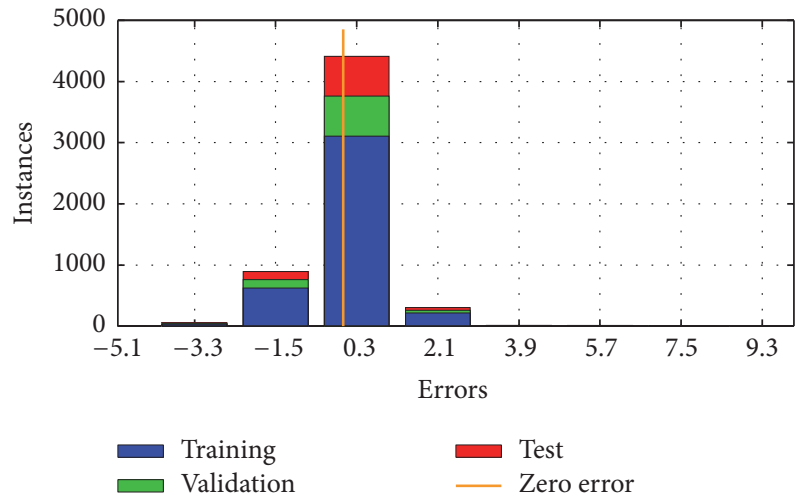

(b)

FIGURE 10: (a) Error diagram of the results from manual feeding for the samples in group 5. (b) Error diagram of predicted result obtained by MSCM-ANN for the samples in group 5.

illustrated by Figure 9, which indicates that the MSCM-ANN is stable to determine the surplus rate and supplemental feeding rate for each group of samples in most of the case. The relatively large deviation of the predicted supplemental feeding rate between training and test for the samples in group 6 may be caused by the large fluctuation of the scrap rate for different orders. Meanwhile, relatively small sample size is harmful to maintain the stability of the model.

Figures 10(a) and 10(b) present the error diagrams of the results obtained from the manual feeding and run predicted results of MSCM-ANN, respectively, for the samples in group 5. Figure 10(b) illustrates that the errors obtained by 


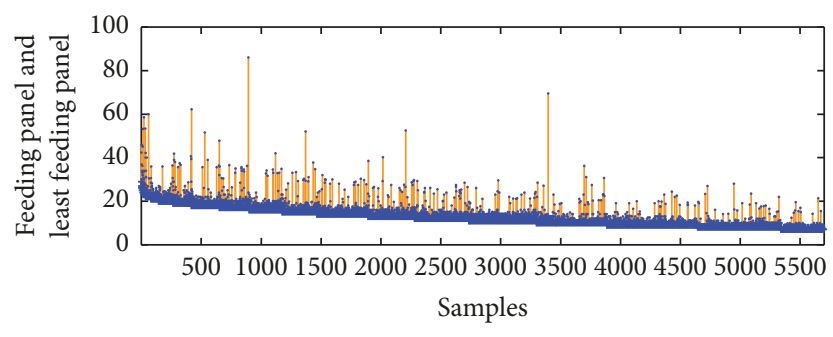

$\begin{array}{ll}\text { - } & \text { Feeding panel } \\ +\quad \text { Least feeding panel } \\ + \text { Surplus panel }\end{array}$

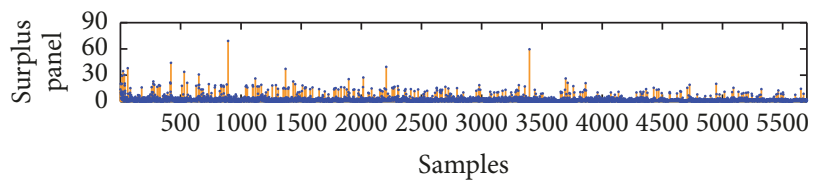

Feeding panel, least feeding panel

(a)

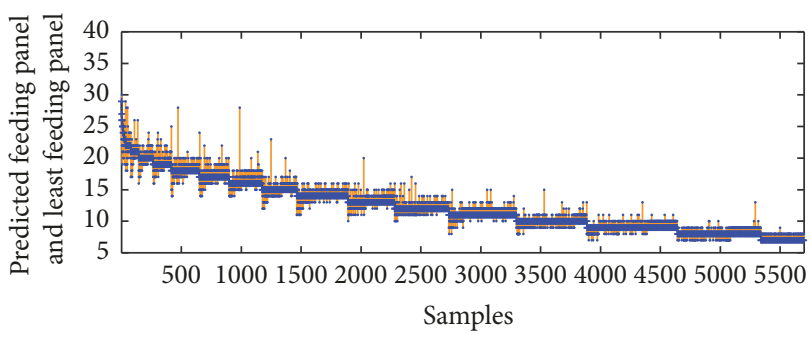

Predicted feeding panel

+ Least feeding panel

_ Predicted surplus panel

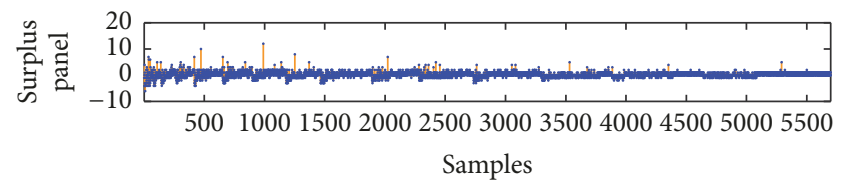

Predicted feeding panel, least feeding panel

(b)

Figure 11: (a) Deviation of samples between manual feeding panel and least feeding panel. (b) Deviation between predicted feeding panel and least feeding panel.

MSCM-ANN are more likely to distribute with mean value 0.3 and short tail for training, validation, and test samples, while most of the errors obtained by the manual feeding distributed with mean value 1.725 (Figure 10(a)), and the large positive tail indicates (Figure 10(a)) that the manual feeding can easily lead to high redundancy after delivery of order. The deviations between the manual feeding panel/predicted feeding panel and least feeding panel for sample in group 5 are illustrated in Figure 11. It indicates that the predicted results in Figure 11(b) achieved lower deviation in most of the case compared to the manual feeding results in Figure 11(a), which can bring lower surplus panel, and therefore reduce the cost of material, production, inventory, and disposal.

Figures 12(a) and 12(b) present the regression of manual feeding panel and predicted feeding panel versus least feeding panel, respectively. Results indicate that the predicted feeding panel coincides better with the least feeding panel in Figure 12(b) compared to the manual feeding panel illustrated in Figure 12(a), and therefore the waste of surplus quantity and area can be reduced. The same coefficients and similar regression expressions obtained by MSCM-ANN for training, validation, test, and all samples mutually verify the stability of the proposed approach.

\section{Conclusions}

Accurate determination of the number of feeding panels for each PCB template order can reduce the cost of material, production, logistics, inventory, disposal, and delivery tardiness compensation. In this paper, a data mining approach (MSCM-ANN) involving the use of multivariate boxplot, MSCM, NCFS, and ANN was developed for establishing the scrap rate prediction model and material feeding optimization for PCB template order considering the structural change influence for the predicted scrap rate. The various aspects of the approaches have been discussed in detail. Mean squared error, mean absolute error, and mean absolute percentage error, three prediction performance indicators, combined with surplus rate and supplemental feeding rate, two most concerned performances indicators for material optimization in practice, were presented to evaluate the established model. The multivariate boxplot was adopted for scarp rate outlier detection considering the structural changes influence of different input features, while the MSCM was applied to explore the multiple structural changes of the samples and therefore partition the samples into 6 different subgroups. NCFS and ANN were utilized for feature selection and scrap rate prediction model establishment for each group of the samples, respectively. After comparing MSCM-ANN with ANN and the manual feeding, the following conclusions and contributions are highlighted as follows.

(1) The proposed MSCM-ANN shows superior prediction accuracy on training, validation, and test dataset with the lowest MSE, MAE, MAPE, surplus rate, and supplemental feeding rate performance indicators compared to ANN and the manual feeding. MSCM-ANN reduces the surplus rate and supplemental feeding rate from $27.44 \%$ and $17.91 \%$ obtained by the manual feeding to $11.96 \%$ and $11.91 \%$, respectively, but ANN can only reduce them to $15.16 \%$ and $12.69 \%$, respectively. The same coefficients and similar regression expressions of the predicted feeding panel versus the least required panel for training, validation, test, and all samples mutually verify the stability of the proposed MSCM-ANN.

(2) The established model provides a new mechanism based on DM for the material feeding optimization of PCB template production that has seldom been studied according to the best of our knowledge. The application of the developed approach can replace the empirical manual feeding and cut 

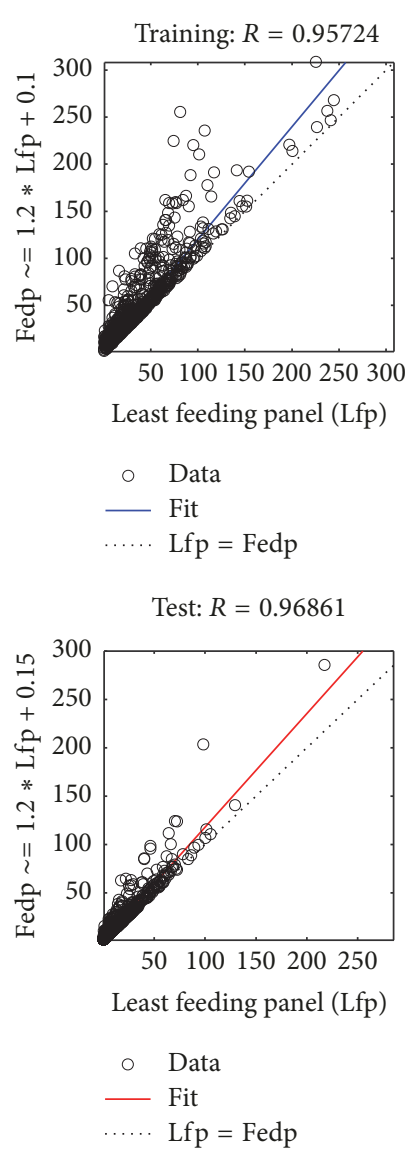

(a)

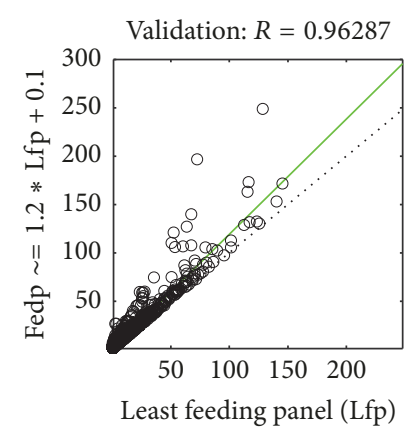

- Data

- Fit

…. Lfp $=$ Fedp

All: $R=0.95945$

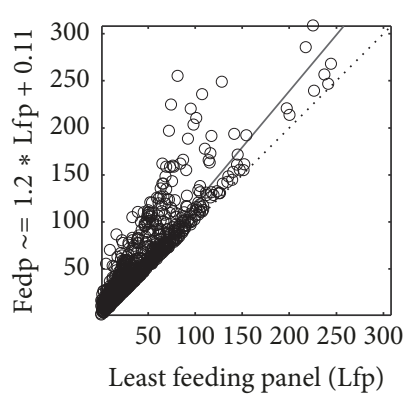

- Data

— Fit

..... Lfp $=$ Fedp

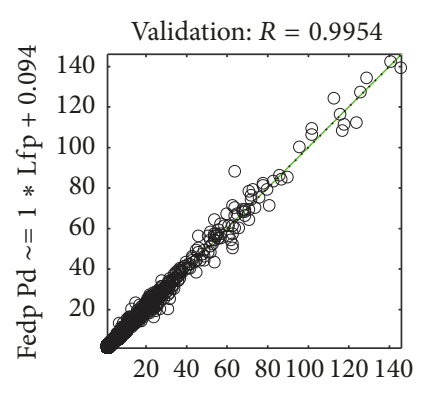

Least feeding panel (Lfp)

- Data

— Fit

..... Lfp $=$ Fedp Pd

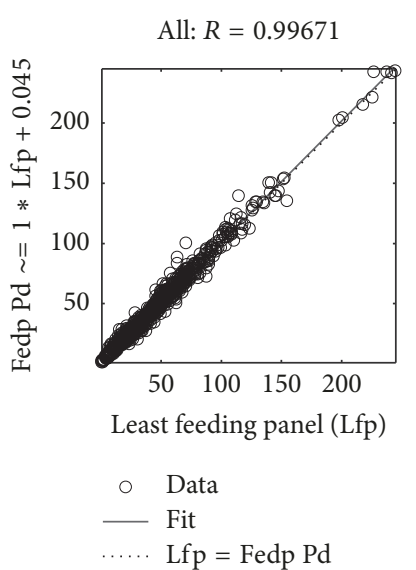

(b)

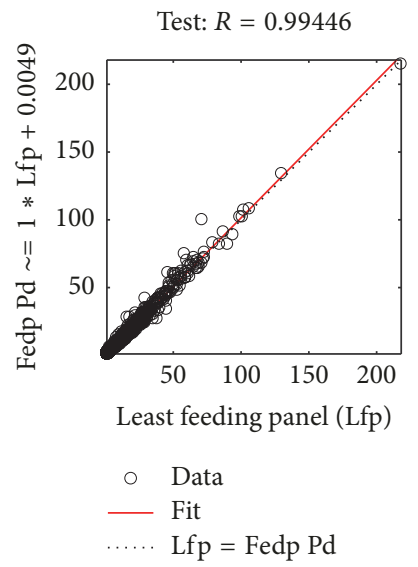

FIGURE 12: (a) Regression of manual feeding panel versus least feeding panel. (b) Regression of predicted feeding panel versus least feeding panel.

down experience dependent workers, but more important it can reduce the comprehensive cost of raw material, production, logistics, inventory, disposal, and delivery tardiness compensation as a whole.

(3) The framework for the material feeding optimization, specified variables, partitioned sample group considering the structural change influence, selected features with mutual verification by different group of the samples, the established ANN-based prediction model, and related transformation can further facilitate other models' development for similar factory.

Tool development based on MSCM-ANN model and practice application should be studied continually. Meanwhile, the structure change of the samples based on other features can be explored and more precise prediction model can also be investigated. The architecture optimization of ANN combined with some heuristic algorithm and the weights updates mechanism considering the balance of surplus and supplemental feeding is being studied by us.

\section{Additional Points}

Featured Application. The research was motivated by the requirement of a printed circuit board (PCB) manufacturing company, and the application of the work is to optimize the material feeding by reducing the surplus rate and supplemental feeding rate simultaneously.

\section{Conflicts of Interest}

The authors declare that they have no conflicts of interest.

\section{Authors' Contributions}

Shengping Lv proposed and implemented the algorithm and also wrote the paper. Binbin Zheng and Qiangsheng Yue conducted the experiments and analyzed the data. Hoyeol Kim proposed the structure of the paper and helped in proofreading and improving the quality of the article.

\section{Acknowledgments}

This paper is supported by the National Natural Science Foundation of China (Grant no. 51605169) and Natural Science Foundation of Guangdong, China (Grant no. 2014A030310345). This study is also supported by the State Scholarship Fund of China (Grant no. 201608440414). The 
authors wish to thank Guangzhou FastPrint Technology Co., Ltd. to provide data for the study.

\section{References}

[1] A. Canal Marques, J.-M. Cabrera, and C. De Fraga Malfatti, "Printed circuit boards: a review on the perspective of sustainability," Journal of Environmental Management, vol. 131, pp. 298306, 2013.

[2] E. Hofmann and M. Rüsch, "Industry 4.0 and the current status as well as future prospects on logistics," Computers in Industry, vol. 89, pp. 23-34, 2017.

[3] R. Cupek, A. Ziebinski, L. Huczala, and H. Erdogan, "Agentbased manufacturing execution systems for short-series production scheduling," Computers in Industry, vol. 82, pp. 245258, 2016.

[4] G. Cheng, "Data and knowledge mining with big data towards smart production," Journal of Industrial Information Integration, 2017.

[5] G. Köksal, İ. Batmaz, and M. C. Testik, "A review of data mining applications for quality improvement in manufacturing industry," Expert Systems with Applications, vol. 38, no. 10, pp. 1344813467, 2011.

[6] D. Delen and H. Demirkan, "Data, information and analytics as services," Decision Support Systems, vol. 55, no. 1, pp. 359-363, 2013.

[7] H. Rostami, J.-Y. Dantan, and L. Homri, "Review of data mining applications for quality assessment in manufacturing industry: support vector machines," International Journal of Metrology and Quality Engineering, vol. 6, no. 4, article 401, 2015.

[8] A. K. Choudhary, J. A. Harding, and M. K. Tiwari, "Data mining in manufacturing: a review based on the kind of knowledge," Journal of Intelligent Manufacturing, vol. 20, no. 5, pp. 501-521, 2009.

[9] P. O’Donovan, K. Leahy, K. Bruton, and D. T. J. O’Sullivan, "Big data in manufacturing: a systematic mapping study," Journal of Big Data, vol. 2, pp. 1-20, 2015.

[10] K. Nagorny, P. Lima-Monteiro, J. Barata, and A. W. Colombo, "Big data analysis in smart manufacturing: a review," International Journal of Communications, Network and System Sciences, vol. 10, no. 03, pp. 31-58, 2017.

[11] T. L. Tseng, C. Johnny, H. Lee, and Y. J. Kwon, "Analysis of complex PCB design data using data mining approach to better estimate the work hours," International Journal of Applied Engineering Research, vol. 11, pp. 11700-11711, 2016.

[12] T.-N. Tsai and M. Liukkonen, "Robust parameter design for the micro-BGA stencil printing process using a fuzzy logic-based Taguchi method," Applied Soft Computing, vol. 48, pp. 124-136, 2016.

[13] T. N. Tsai, "Modeling and optimization of stencil printing operations: a comparison study," Computers \& Industrial Engineering, vol. 54, pp. 374-389, 2008.

[14] N. Khader, S. W. Yoon, and D. Li, "Stencil printing optimization using a hybrid of support vector regression and mixed-integer linear programming," Procedia Manufacturing, vol. 11, pp. 18091817, 2017.

[15] H. Haneda, H. Kodama, T. Hirogaki, E. Aoyama, and K. Ogawa, "Investigation of drilling conditions of printed circuit board based on data mining method from tool catalog data-base," Advanced Materials Research, vol. 939, pp. 547-554, 2014.
[16] P. K. Srimani and V. Prathiba, "Adaptive data mining approach for PCB defect detection and classification," Indian Journal of Science and Technology, vol. 9, no. 44, Article ID 98964, 2016.

[17] H. Sim, D. Choi, and C. O. Kim, "A data mining approach to the causal analysis of product faults in multi-stage PCB manufacturing," International Journal of Precision Engineering and Manufacturing, vol. 15, no. 8, pp. 1563-1573, 2014.

[18] E. Hadavandi, H. Shavandi, and A. Ghanbari, "An improved sales forecasting approach by the integration of genetic fuzzy systems and data clustering: case study of printed circuit board," Expert Systems with Applications, vol. 38, no. 8, pp. 9392-9399, 2011.

[19] P.-C. Chang, C.-H. Liu, and C.-Y. Fan, "Data clustering and fuzzy neural network for sales forecasting: a case study in printed circuit board industry," Knowledge-Based Systems, vol. 22, no. 5, pp. 344-355, 2009.

[20] P.-C. Chang, C.-H. Liu, and R. K. Lai, "A fuzzy case-based reasoning model for sales forecasting in print circuit board industries," Expert Systems with Applications, vol. 34, no. 3, pp. 2049-2058, 2008.

[21] P.-C. Chang, Y.-W. Wang, and C.-H. Liu, "The development of a weighted evolving fuzzy neural network for PCB sales forecasting," Expert Systems with Applications, vol. 32, no. 1, pp. 86-96, 2007.

[22] A. Tavakkoli, J. Rezaeenour, and E. Hadavandi, "A novel forecasting model based on support vector regression and bat meta-heuristic (Bat-SVR): case study in printed circuit board industry," International Journal of Information Technology \& Decision Making, vol. 14, no. 1, pp. 195-215, 2015.

[23] V. J. Hodge and J. Austin, "A survey of outlier detection methodologies," Artificial Intelligence Review, vol. 22, no. 2, pp. 85-126, 2004.

[24] A. Zimek, E. Schubert, and H.-P. Kriegel, "A survey on unsupervised outlier detection in high-dimensional numerical data," Statistical Analysis and Data Mining, vol. 5, no. 5, pp. 363-387, 2012.

[25] J. Bai and P. Perron, "Estimating and testing linear models with multiple structural changes," Econometrica, vol. 66, no. 1, pp. 4778, 1998.

[26] J. Bai and P. Perron, "Computation and analysis of multiple structural change models," Journal of Applied Econometrics, vol. 18, no. 1, pp. 1-22, 2003.

[27] Z. Qu, "Estimating and testing structural changes in multivariate regressions," 2017, http://people.bu.edu/perron/code.html.

[28] W. Yang, K. Wang, and W. Zuo, "Neighborhood component feature selection for high-dimensional data," Journal of Computers, vol. 7, no. 1, pp. 162-168, 2012.

[29] V. Bolón-Canedo, N. Sánchez-Maroño, and A. AlonsoBetanzos, "A review of feature selection methods on synthetic data," Knowledge and Information Systems, vol. 34, no. 3, pp. 483-519, 2013.

[30] G. Chandrashekar and F. Sahin, "A survey on feature selection methods," Computers \& Electrical Engineering, vol. 40, no. 1, pp. 16-28, 2014.

[31] G. Aldehim and W. Wang, "Determining appropriate approaches for using data in feature selection," International Journal of Machine Learning and Cybernetics, vol. 8, no. 3, pp. 915-928, 2017.

[32] "Mathworks," 2017, https://www.mathworks.com/help/stats/ neighborhood-component-analysis.html. 
[33] “Artificial neural network," 2017, https://en.wikipedia.org/wiki/ Artificial neural_network\&gt.

[34] S. T. Hashemi, O. M. Ebadati E., and H. Kaur, "A hybrid conceptual cost estimating model using ANN and GA for power plant projects," Neural Computing and Applications, 2017.

[35] G. Wang, T. Xu, T. Tang, T. Yuan, and H. Wang, "A Bayesian network model for prediction of weather-related failures in railway turnout systems," Expert Systems with Applications, vol. 69, pp. 247-256, 2017. 


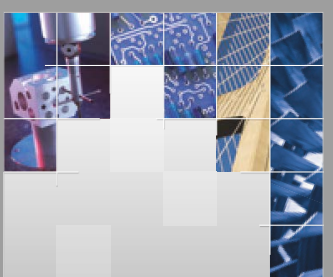

\section{Enfincering}
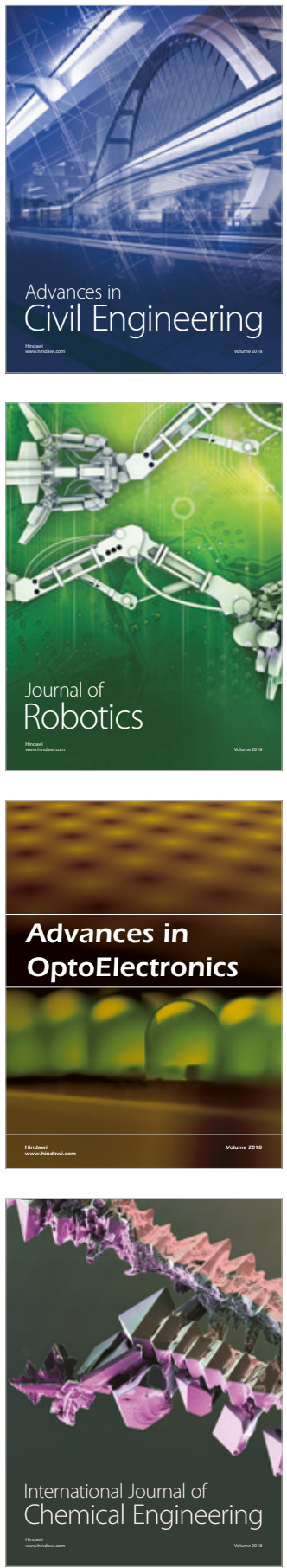

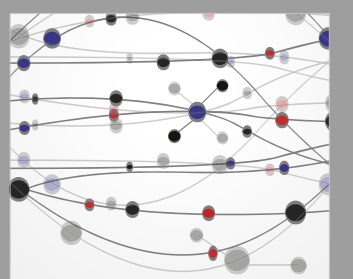

\section{Rotating \\ Machinery}

The Scientific World Journal

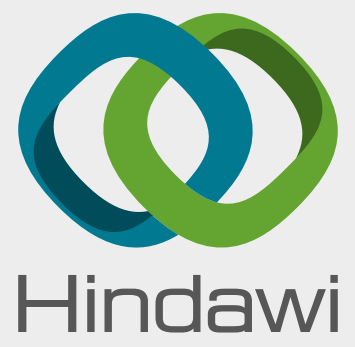

Submit your manuscripts at

www.hindawi.com
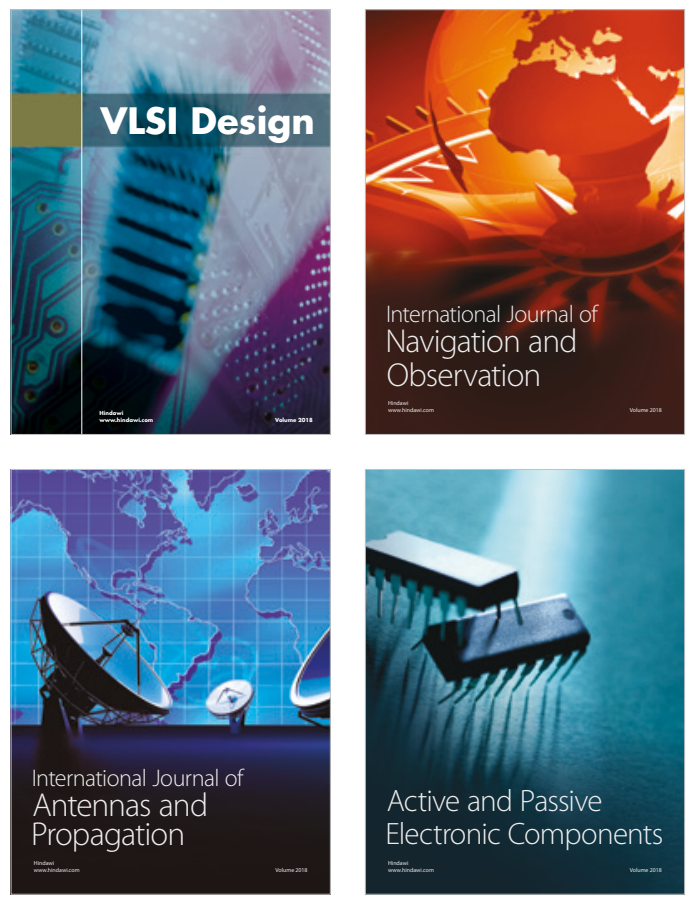
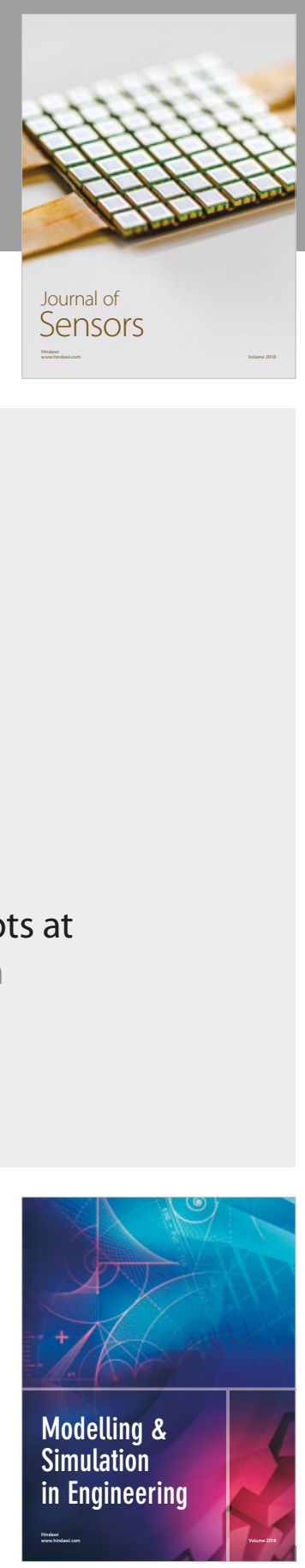

\section{Advances \\ Multimedia}
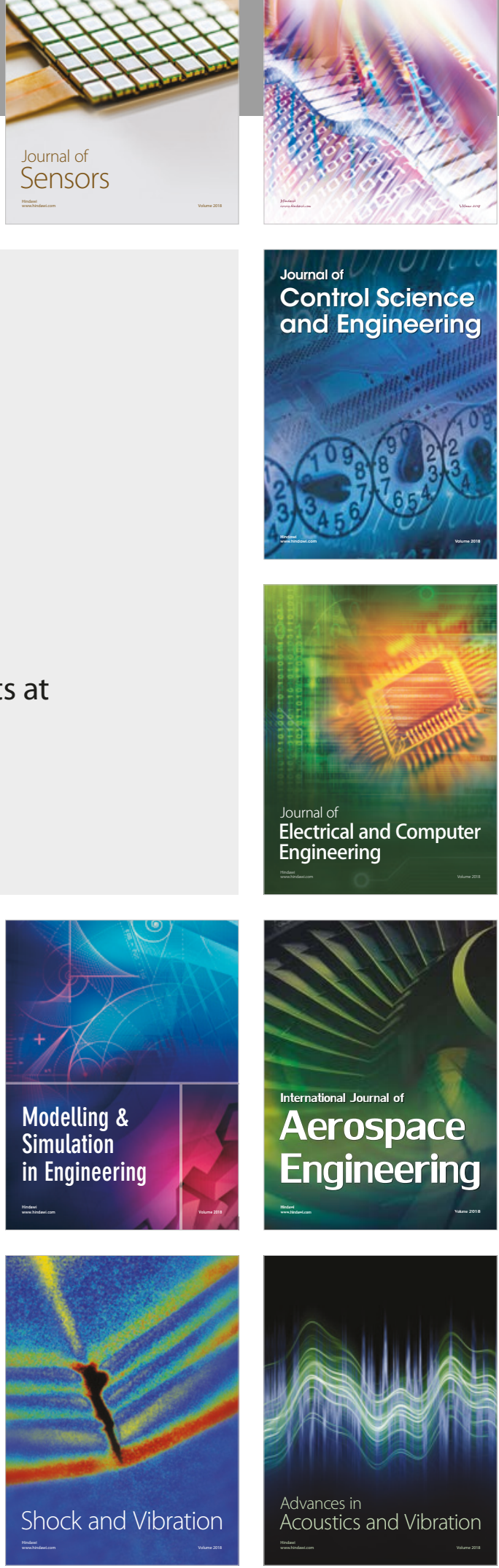